\title{
Surgical Treatment of Humeral Shaft Fractures by Arthroscopy- assisted Intramedullary Nail Fixation
}

\author{
(D) Ali Yüce1, (D) Yunus İmren², (D) Süleyman Semih Dedeoğlu², (1D) Müjdat Adaş², (D) Cem Dinçay Büyükkurt2, \\ (D) Tahsin Olgun Bayraktar³, (D) Hakan Gürbüz² \\ 1Sarıkamış State Hospital, Clinic of Orthopaedics and Traumatology, Kars, Turkey \\ 2University of Health Sciences Turkey, Prof. Dr. Cemil Taşçığlu City Hospital, Clinic of Orthopaedics and Traumatology, İstanbul, Turkey \\ ${ }^{3}$ Bahçelievler State Hospital, Clinic of Orthopaedics and Traumatology, İstanbul, Turkey
}

\section{Abstract}

Objective: Humeral shaft fractures face with controversies about the entry site of intramedullary nailing (IMN) due to its potential complications. We aimed to evaluate feasibility of arthroscopy-assisted intramedullary nail fixation for the management of these fractures with respect to its mid-term clinical and functional outcomes, complication rates of nail entry site, and fluoroscopy duration.

Methods: We examined medical data of 21 patients who underwent arthroscopy-assisted IMN after closed reduction upon diagnosis of humeral shaft fracture. Analyzed parameters included duration of anesthesia, surgery, fluoroscopy, preoperative hospitalization, and union; surgical complication rates; and the degree of lateralization between ideal and applied nail entry site, as measured by shoulder computed tomography scans. Shoulder functions were assessed by Constant and American Shoulder and Elbow Surgeons (ASES) scoring.

Results: Patients were found to be followed up for a mean of $22.8 \pm 4.76$ months. The mean duration of surgery was $56.9 \pm 14.27$ minutes. The mean fluoroscopy time was detected as $1.63 \pm 0.49$ minutes. Distal locking was performed without fluoroscopy (electromagnetic targeting or internal locking nail) in 15 patients and with free hand method under fluoroscopy in six patients. The mean duration of fluoroscopy in these techniques were $1.47 \pm 0.41$ minutes and $1.91 \pm 0.52$ minutes, respectively. No patient was found to have subacromial impingement syndrome. The mean lateralization of nail entry sites was measured as $0.61 \pm 0.73 \mathrm{~mm}$. The mean degree of varus was $2.38 \pm 1.18$. The mean ASES and Constant scores were found as $89 \pm 2.81$ and $90 \pm 4.59$ points,respectively. Excellent/good functional outcomes had been reported by $90.5 \%$ of the study population.

Conclusion: Arthroscopy-assisted technique may minimize rotator cuff injury and thereby may provide satisfactory outcomes in postoperative shoulder functions. This technique may be a feasible and safe option associated with reduced entry site complications and potentially less exposure to radiation from shorter use of fluoroscopy.

Keywords: Humerus, shaft, fracture, arthroscopy-assisted, intramedullary nail, antegrade

\section{INTRODUCTION}

Humeral shaft fractures constitute $5-8 \%$ of all extremity fractures with an annual incidence of 13/100.000. These fractures are managed with either conservative or surgical approaches (1). While the former includes functional bracing, spica cast, Velpeau bandage, and coaptation splint; surgical approaches are platescrew fixation, intramedullary nailing, and external fixator $(1,2)$.
In humeral fractures, antegrade intramedullary nailing (IMN) has the advantages of minimally invasive fashion, rapid fracture healing, lower iatrogenic radial nerve damage. On the other hand, it might also cause long-term shoulder dysfunction after injury to rotator cuff and cartilage at the entry site. The fact that the proximal nail may stay within the joint could impair shoulder functions due to subacromial impingement and rotator cuff
Address for Correspondence: Ali Yüce, Sarıkamış State Hospital, Clinic of Orthopaedics and Traumatology, Kars, Turkey

Phone: +90 5425529858 E-mail: dr_aliyuce@hotmail.com ORCID ID: orcid.org/0000-0003-3751-6644

Cite this article as: Yüce A, Imren Y, Dedeoğlu SS, Adaș M, Büyükkurt CD, Bayraktar TO, Gürbüz H. Surgical Treatment of Humeral Shaft Fractures by Arthroscopy-assisted Intramedullary Nail Fixation. Eur Arch Med Res 2020; 36 (2):108-14

๑Copyright 2020 by the University of Health Sciences Turkey, Prof. Dr. Cemil Taş̧ıŏlu City Hospital European Archives of Medical Research published by Galenos Publishing House.
Received: 28.06 .2019 Accepted: 15.10 .2019 
irritation, increasing the need for nail removal. Other drawbacks of intramedullary nails include iatrogenic fractures during IMN and radiation exposure due to fluoroscopy (3-5).

In this study, we aimed to evaluate feasibility of the arthroscopyassisted IMN of the humerus in terms of entry site complications and duration of fluoroscopy.

\section{METHODS}

After obtaining approval from local ethics committee (Okmeydanı Training and Research Hospital 19.12.2017-789), medical records of 21 patients who underwent arthroscopy-assisted antegrade humeral IMN fixation upon surgical indication due to the diagnosis of humeral shaft fracture between January 2015 and September 2017 were retrospectively reviewed in this study. Informed consent was obtained from all individual participants included in the study. Included patients had to have clinical and radiological documentation of humeral diaphyseal fracture where the surgery was indicated with following criteria: shortening of $>3 \mathrm{~cm},>30^{\circ}$ angulation at coronal plane, and $>20^{\circ}$ angulation at sagittal plane. Patients with open epiphyseal lines at the proximal humerus, infected fractures, GustiloAnderson type 3 open fractures, multiple fractures, and fractures where open reduction was performed were excluded.

Medical data of the patients were collected by reviewing medical records, operating room records, arthroscopy and fluoroscopy image recordings, plane X-ray and computed tomography images taken during outpatient visits, and physician- or patientreported shoulder functions as assessed by Constant and ASES scores.
Implanted nails were belonged to two different companies. Fourteen patients had been implanted Trigen humeral nail of Smith and Nephew. Six of these patients underwent nailing by distal locking under fluoroscopic guidance with free hand method. For the remaining eight patients, distal locking was performed without fluoroscopy by using electromagnetic targeting (Smith and Nephew-Sureshot). Seven patients had been implanted TST's InsafeLock humerus nail. Distal locking of this nail was performed via using elastic, threaded pin system which was advanced through the nail. Fluoroscopy was not used for distal locking in these patients.

\section{Surgical Technique}

Intravenous cefazolin prophylaxis was administered for a total of 24 hours with the initial dose one hour before the surgery. All patients were prepared in lawn chair position under general anesthesia. Fluoroscopy device and arthroscopy tower were positioned to be suitable for surgery. Following the essential sterilization and covering procedures, anatomic markers and fracture line were determined with marker pen on the side to be operated. The surgery was started with the incision performed through the inferomedial and posterior of the lateral edges of the spine of scapula, through which posterior arthroscopic port was inserted to visualize the joint. In order to determine the entry site of the nail, a $2.2 \mathrm{~mm}$ Kirschner wire was introduced percutaneously over the joint cartilage about $20 \mathrm{~mm}$ medial to the greater tubercle posterior to the biceps tendon $1.5 \mathrm{~cm}$ distal to the anterolateral border of the acromion) under the guidance of fluoroscopy, targeting humeral shaft and intramedullary cavity (Figure 1).

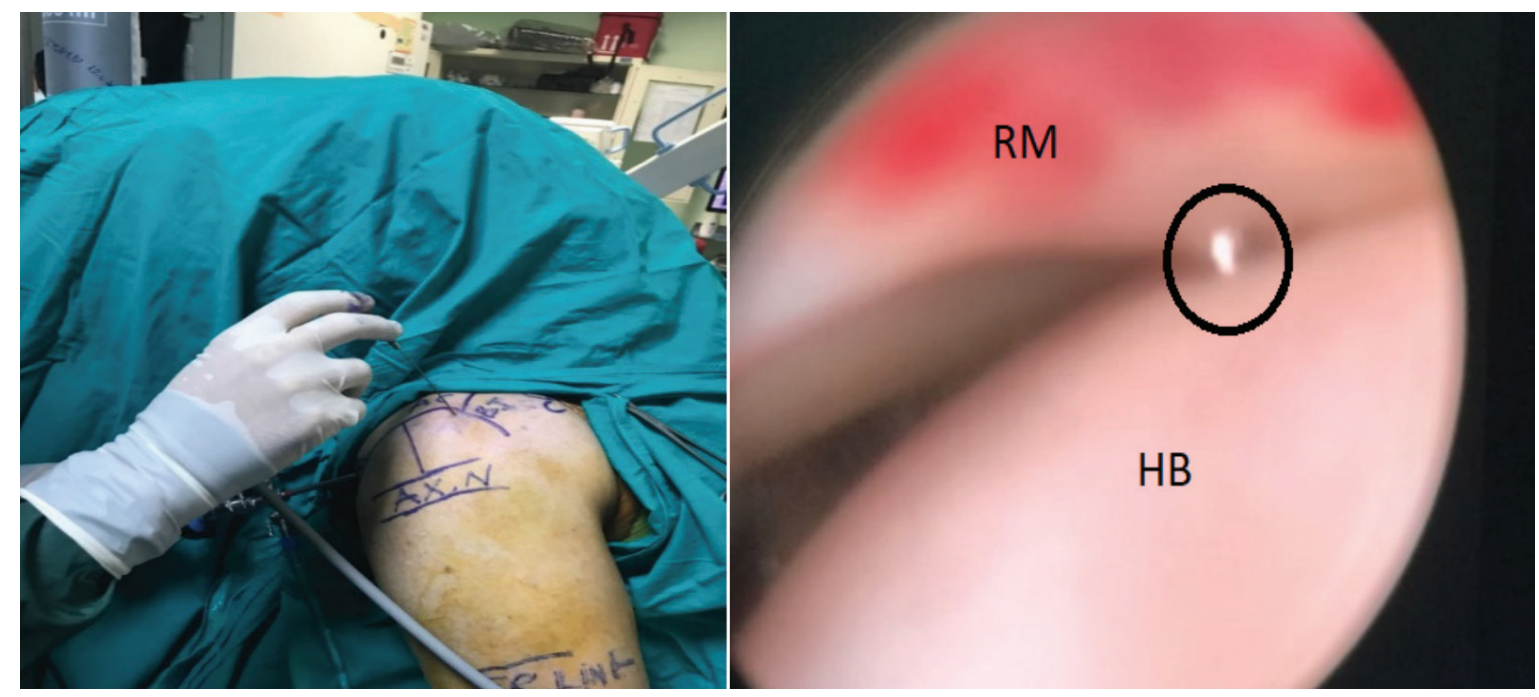

Figure 1. Percutaneous insertion of the guidewire after identifying the entry site (left) and arthroscopic image of the inserted guidewire to the entry site (right)

HB: Humeral head, RM: Rotator cuff 
After arthroscopic and fluoroscopic verification, a mini skin incision of approximately $2 \mathrm{~cm}$ was made at the level of the K-wire. Deltoid fibers were passed through blunt dissection to reach at the supraspinatus muscle. Supraspinatus muscle was separated by a $10 \mathrm{~mm}$ incision which was parallel to its tendon's extension. A cannulated drill was sent over K-wire to the proximal humerus. One guidewire was sent from the drilled part to pass through the fracture line. After fracture reduction was confirmed under fluoroscopy; humeral medulla was reamed with flexiblebendable reamers. Afterwards, intramedullary humeral nail was inserted in appropriate diameter and length.

After arthroscopically ensuring that the proximal part of the nail was embedded to the joint cartilage, not leading to subacromial impingement, the proximal nail was locked by two screws delivered over the external guide. The heads of these screws were arthroscopically confirmed not to penetrate the joint cartilage (Figure 2A). In patients where internal locking nail had not been used, distal locking was performed with two screws via mini incision (with or without fluoroscopy depending on the brand/model of the nails used). Top screw was inserted under arthroscopic guidance. Finally, it was ensured that the proximal part of the nail was not within the joint cavity (Figure 2B). Afterwards, supraspinatus muscle was repaired with an unabsorbable suture followed by suturing of the skin. The joint was irrigated through arthroscopic port to eliminate intraarticular debris before the arthroscopic entry site was closed with unabsorbable suture, and the surgery was terminated.

\section{Statistical Analysis}

The suitability of the data to the normal distribution was tested with Shaphiro-Wilk test, Student's t-test was used for comparison of features with normal distribution in 2 independent groups, and Mann-Whitney $U$ test was used for comparison of normal distribution with 2 independent groups. The variables with categorical measurements were analyzed by Pearson and Fisher's exact chi-square tests. Descriptive statistics are given as mean \pm standard deviation for numerical variables and number and \% values for categorical variables. SPSS Windows version 24.0 package program was used for statistical analysis and $p<0.05$ was considered statistically significant.

\section{RESULTS}

The study included 11 male and 10 female patients. The mean age was found to be $70.66 \pm 17.5$ years. The mean follow-up period was $22.8 \pm 4.76$ months. The mechanisms of injury showed the most common etiology to be the fractures occurring after simple fall in 13 patients. One patient (4.7\%) had preoperative radial nerve deficit which was improved during the follow-up. One patient had superficial wound infection in the postoperative period and treated with intravenous antibiotics.

The fractures of the patients were grouped according to AO fracture classification, where four different fracture types were determined. The distribution of the groups was determined as nine patients for $\mathrm{A} 1$, six patients for $\mathrm{A} 2$, and three patients for each of $\mathrm{A} 3$ and $\mathrm{B} 1$.

The mean preoperative duration was detected as $3.19 \pm 1.83$ days. The mean duration of the surgery and anesthesia was $56.9 \pm 14.27$ minutes and $82.57 \pm 14.44$ minutes, respectively. The mean time for union was found as $18.12 \pm 3.01$ weeks.
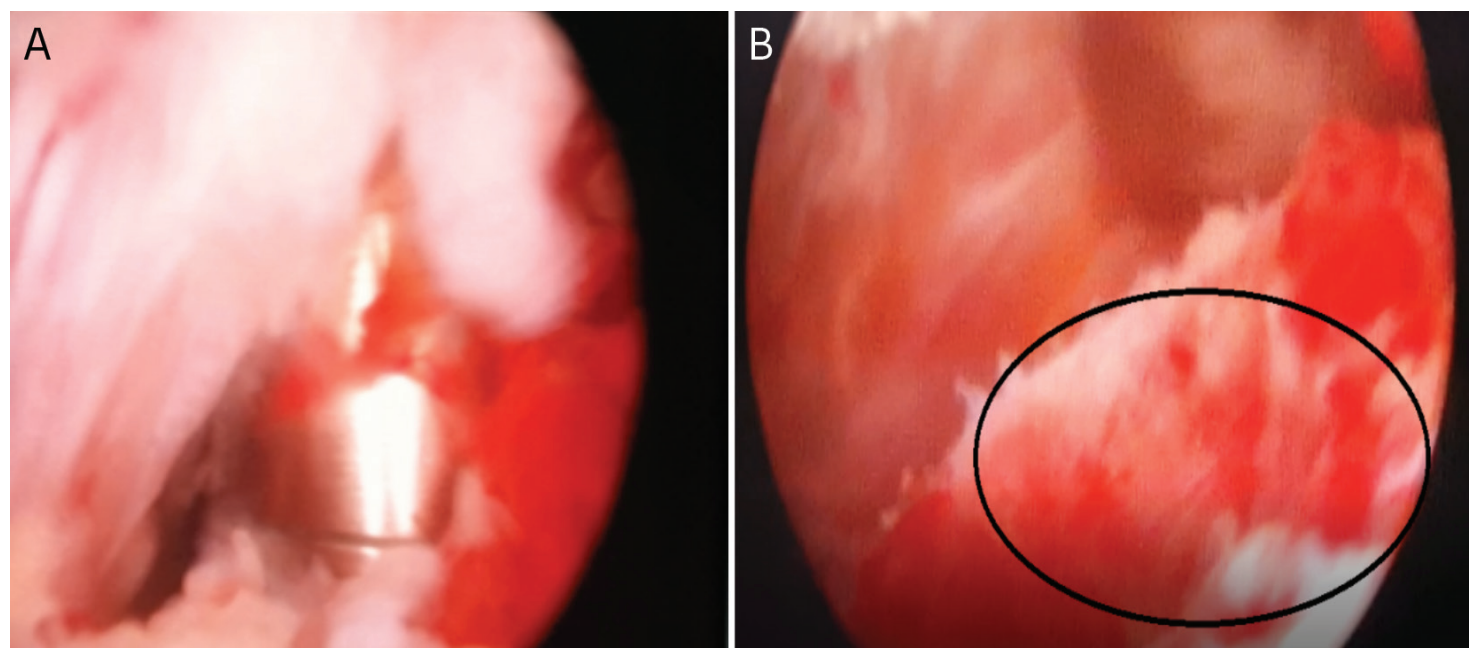

Figure 2. A) In arthroscopic view, the nail was inserted into the humerus. The line on the image belonged to the external guide, showing that the nail was completely within the joint (left). B) The black ring showed the entry site of the nail postoperatively, with the nail completely inside the joint, in arthroscopic view (right) 
One patient was found to be operated due to delayed union after conservative approach. Twenty patients underwent surgery due to primary fracture. Distal locking was performed without fluoroscopy in 15 patients and with free hand method under fluoroscopy in six patients. The mean duration of fluoroscopy in these techniques were $1.47 \pm 0.41$ minutes and $1.91 \pm 0.52$ minutes, respectively; while the overall duration of fluoroscopy was $1.63 \pm 0.49$ minutes.

No patient was found to have subacromial impingement syndrome due to positioning of the proximal nail superior to the level of cartilage surface. The mean length of lateralization between ideal and applied nail entry site, as measured by shoulder computed tomography axial images, was $0.61 \pm 0.73$ $\mathrm{mm}$. No patient was found to develop valgus deformity after reduction. The mean degree of varus was $2.38^{\circ} \pm 1.18^{\circ}$. The mean antero-posterior angulation was $2.54^{\circ} \pm 1.22^{\circ}$. The mean ASES and Constant scores were found as $89 \pm 2.81$ and $90 \pm 4.59$ points, respectively. Constant scoring was detected to reveal excellent/ good functional outcomes in $90.5 \%$ of the study population.

The effects of the distal locking performed with free hand method or without fluoroscopy on total fluoroscopy time were also compared. There was no significant difference between the two methods $(p=0.107)$. There was also no statistically significant difference between distal locking methods in terms of the duration of the surgery $(p=0.482)$. The mean time of fluoroscopy was determined to be longer in $\mathrm{AO}$ type $\mathrm{A} 3$ fractures than that in A0 type A1, type A2, and type B1 fractures. The association between the mean length of lateralization of the entry site and the mean degree of postoperative varus was found as statistically significant $(p=0.005)$ and Pearson test showed positive correlation $(r=0.351)$, (Figure 3 ).

\section{DISCUSSION}

A major disadvantage of the intramedullary nails is the need for fluoroscopy, which poses a risk for surgeons and other operating room staff during the surgical procedure (6). Mean times of fluoroscopy used in IMN vary between 2-4.6 minutes in humeral fractures (6-8). Franck et al. (9) in their study with expandable humerus nailing, reported the mean duration of fluoroscopy to be 1.5 minutes, concluding it to be very short while proximal and distal locking were not performed. In our study, the mean duration of fluoroscopy used during the surgery was 1.63 minutes. In order to determine nail entry site, to drill its proximal part, and to ensure whether proximal nail and proximal locking screws penetrate the joint during arthroscopic-assisted humeral nailing, using a camera might decrease the need for fluoroscopy.

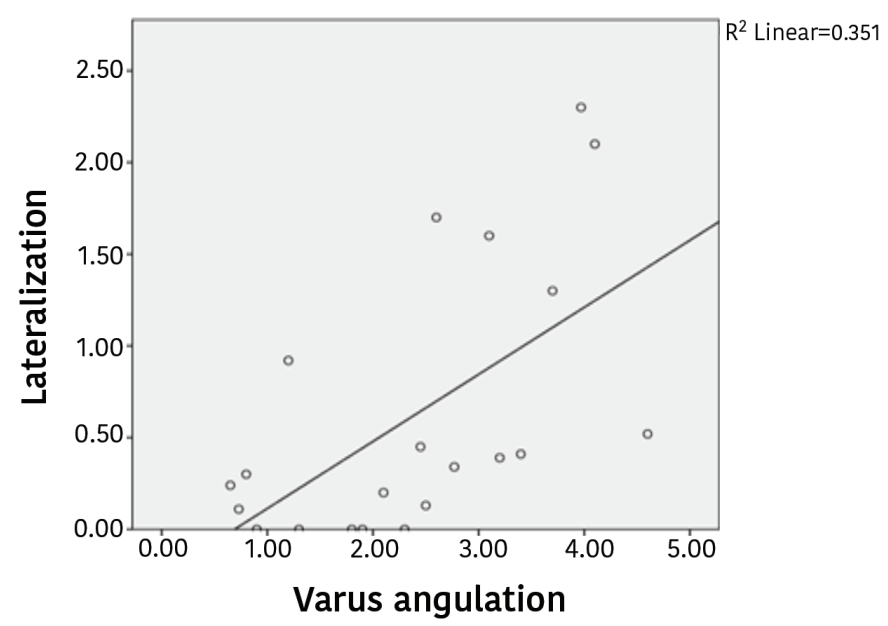

Figure 3. The association between the degree of postoperative varus and the length of lateralization of the nail on CT axial images

CT: Computed tomography

In this context, arthroscopy-assisted IMN may be associated with reduced radiation exposure to the operating room staff and the patient.

Fluoroscopy-guided locking techniques applied during distal locking of intramedullary nails also substantially prolong the surgery time and increase the amount of radiation being exposed. The duration of fluoroscopy can be reduced by using electromagnetic targeting during distal locking (10). The mean fluoroscopy time of 15 patients to whom distal locking was performed without fluoroscopy was 1.47 minutes, compared to that of 1.91 minutes in those to whom distal locking was performed with free hand method; where no significant difference was found. On the other hand, AO types A3 fractures were determined to have significantly prolonged fluoroscopy than that in other types of fractures. We partly attribute the lack of the differential effect of distal locking methods on fluoroscopy time to potential two factors. First, the failure to record the fluoroscopy duration in those where only distal locking was performed did not allow us to make a statistical comparison in fluoroscopy time. In addition, fracture types influenced the fluoroscopy time in varying degrees. We suggest that incorporation of fluoroscopy-free distal locking to the arthroscopy-assisted IMN could further reduce radiation exposure.

Several studies reported mean duration of surgery in antegrade humerus nailing to vary between 50.8 to 78 minutes (2,3,6$8,11,12)$. In our study, we determined that the average duration of surgery was 56.9 minutes. It might be suggested that arthroscopy, as an additional procedure to standard antegrade nailing, may not be associated with prolonged operation time during arthroscopy-assisted IMN. 
The incidence of iatrogenic greater tubercle fractures during antegrade nailing is $2-11 \%$. It occurs when the entry site of the rigid nail remains at the lateral. During nailing, while the tip of the nail is based on the medial cortex of the humerus, the nail makes a pressure on the lateral cortex of the humerus due to the leveraging effect of the acromion; which may result in fractures of the greater tubercle $(3,8)$. In our study, no iatrogenic tubercle fracture was detected. Mean length of lateralization between the ideal and applied nail was measured as $0.61 \mathrm{~mm}$, where the nails appear to be inserted to the proper position with a likely negligible deviation. Arthroscopic technique may provide the benefit of accurate identification of the entry site by direct visualization, which might be associated with the prevention of intraoperative greater tubercle fractures.

IMN of the humerus may be complicated with varying degrees of varus angulation that may cause functional impairment (3). Primary malalignment is largely the result of an unsuitable entry site or a fracture site malreduction (13). A nail insertion from the correct entry point should automatically ensure cortical alignment of the fracture (14). In our study, anteroposterior and lateral radiographs of the patients did not show valgus. On the other hand, angulations found at coronal $\left(2.38^{\circ}\right)$ and sagittal $\left(2.54^{\circ}\right)$ planes could be regarded as negligible in cosmetic and functional aspects. This could be explained by correct positioning of the nails to entry sites in all patients. Arthroscopy-assisted IMN might be associated with reduced rates of primary malalignment due to the inappropriate nail entry sites (Figure 4).

The duration of union after antegrade nailing ranged between 6.7 weeks to 13.9 weeks $(2,6,15)$. In our study, the mean union time was $18.12 \pm 3.01$ weeks, which seem to be several weeks longer than those reported in the literature. In our study, 61.9\% of the patients had fractures after simple falls and the mean age was 70.66 years. We attribute prolonged union detected in our study partly to advanced age and osteoporosis.

Remaining of the proximal of the nail under or within the rotator cuff has been associated with several complications, including shoulder pain and subacromial impingement $(3,16)$. Baltov et al. (3) reported shoulder complaints and delay in resuming shoulder and usual arm functions in $7.2 \%$ of their cases secondary to positioning of the proximal nail within the joint. They also reported subacromial impingement due to insufficient embedment of the nail in $10.8 \%$ of patients, and shoulder problems due to compression caused by the head of the proximal locking screw in $4.5 \%$. The authors also reported the need for additional surgical procedures for these problems. In our study, no proximal part or proximal locking screw of the nail was within the joint. We believe that arthroscopy-assisted IMN may be associated with reduced rates of the subacromial impingement owing to direct joint visualization.

Fan et al. (2) reported mean Constant score as 90.2 and ASES score as 90.4 in their IMN patients. Campochiaro et al. (17) reported mean Constant score as 90.6 points, which they suggested as very satisfactory. Rajagopal et al. (18) found mean Constant score as 90.5 points, and reported excellent-good outcomes in 95\% of their patients. The mean ASES score was reported 93.3 points by these investigators. Several factors could yield better should functions by reducing rotator cuff-related problems and iatrogenic shoulder pain: use of a medial entry site away from the avascular region of the rotator cuff, a delicate surgical dissection, a thorough embedment of the nail into the humerus, gentle progressive drilling, correct entry point, minimal injury to the rotator cuff, proper insertion of the nail tip, and removal of
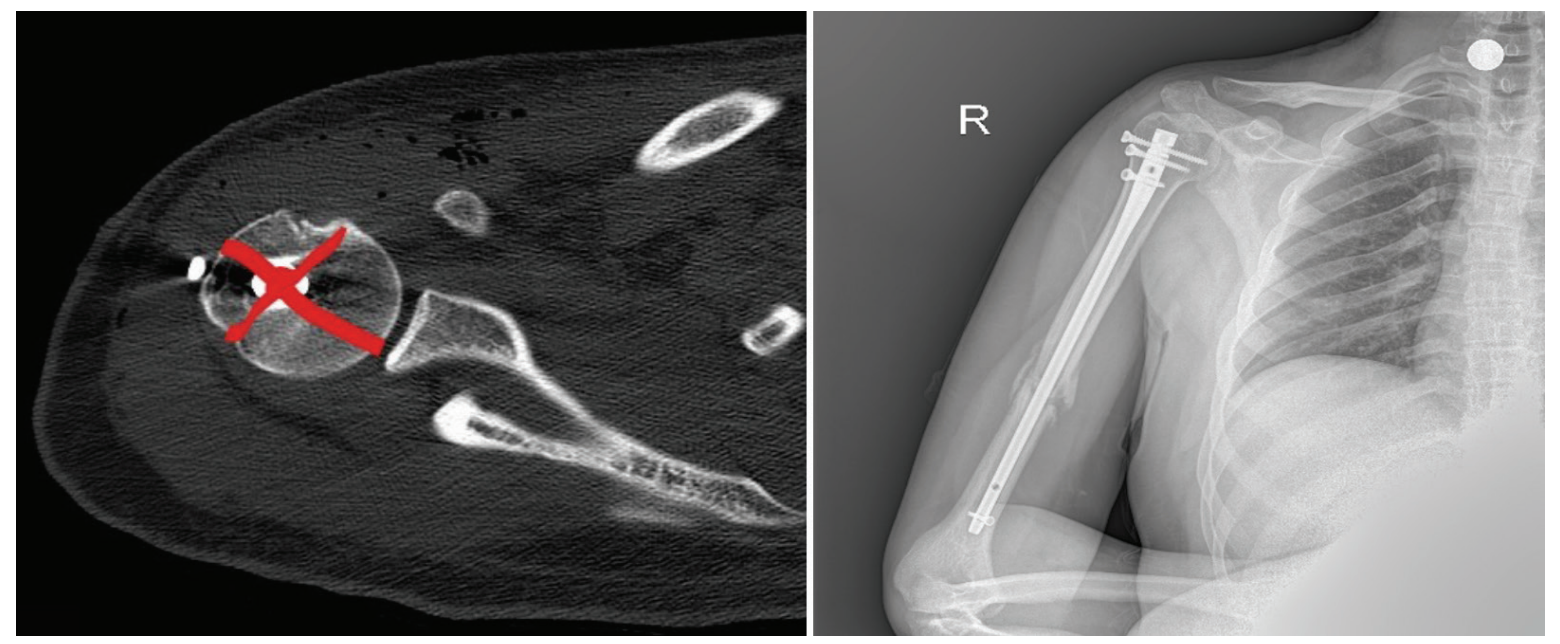

Figure 4. Axial section of the computed tomography of a patient in the postoperative period showing that the nail is at the appropriate insertion point, marked with an "X" (left). The postoperative X-ray of the same patient, where the varus was measured as $0.63^{\circ}$ (right) 
the intraarticular debris $(18,19)$. In our study, the mean Constant score was 90 points and $90.5 \%$ of patients had excellent-good functional outcomes. On the other hand, the mean ASES score was 89. In light of these findings, arthroscopy-assisted IMN could provide several advantages such as reduced rotator cuff injury during humeral drilling and nail insertion, accurate entry point, irrigation and removal of intraarticular debris postoperatively, and lack of rotator cuff irritation by the nail; which might be associated with sufficient level of shoulder functions and reduced need for physical therapy postoperatively.

Axillary nerve deficit due to long proximal skin incision is also a rare complication of antegrade nailing reported in the literature (20). In our study, no axillary nerve damage was observed. The surgical incision in arthroscopy-assisted IMN is short enough for the nail to pass and ends far away from the axillary nerve level, which could prevent against any potential axillary nerve injury.

Arthroscopy has been reported have several complications, including the risk of intraarticular and extraarticular infections, respiratory problems due to fluid extravasation, skin necrosis, neurovascular damage, iatrogenic tendon and deltoid injury, shoulder rigidity, and chondrolysis (21-23). In our study, we did not detect any complications related to shoulder arthroscopy. Considering that arthroscopy-assisted IMN is a combined procedure that incorporates antegrade humeral nailing and shoulder arthroscopy, the possibility of the complications pertaining to the latter will always exist, which might be regarded as a drawback.

The shortcomings of the study were retrospective and the fact that arthroscopy-assisted nailing could not be compared with standard antegrade intramedullary nailing. In addition, the only missing part is that the fluoroscopy times cannot be recorded and compared during distal locking.

\section{CONCLUSION}

It appears that arthroscopy-assisted technique has the potential to minimize rotator cuff injury compared to the conventional antegrade approach, which in turn, provides satisfactory outcomes in postoperative shoulder functions. It may further reduce entry site complication rates and radiation exposure from the use of fluoroscopy. Therefore, we believe arthroscopyassisted IMN to be a feasible and safe method for humeral shaft fractures.

\section{Ethics}

Ethics Committee Approval: Okmeydanı Training and Research Hospital 19.12.2017-789.
Informed Consent: Informed consent was obtained from all individual participants included in the study.

Peer-review: Externally peer-reviewed.

\section{Authorship Contributions}

Concept: H.G., Y.I., A.Y., Design: A.Y., C.D.B., Analysis or Interpretation: A.Y., H.G., Y.I., Literature Search: A.Y., C.D.B., T.O.B., Writing: A.Y., S.S.D., M.A.

Conflict of Interest: No conflict of interest was declared by the authors.

Financial Disclosure: The authors declared that this study received no financial support.

\section{REFERENCES}

1. Updegrove GF, Mourad W, Abboud JA. Humeral Shaft Fractures. J Shoulder Elbow Surg 2018;27:87-97.

2. Fan Y, Li YW, Zhang HB, Liu JF, Han XM, Chang X, et al. Management of humeral shaft fractures with intramedullary interlocking nail versus locking compression plate. Orthopedics 2015;38:825-9.

3. Baltov A, Mihail R, Dian E. Complications after interlocking intramedullary nailing of humeral shaft fractures. Injury 2014;45(Suppl 1):9-15

4. Pogliacomi F, Devecchi A, Costantino C, Vaienti E. Functional long-term outcome of the shoulder after antegrade intrame-dullary nailing in humeraldiaphyseal fractures. Chir Organi Mov 2008;92:11-6.

5. Hohmann E, Glatt V, Tetsworth K. Response to letter to the editor regarding "Minimally invasive plating versus either open reduction and plate fixation or intramedullary nailing of humeral shaft fractures: a systematic review and meta-analysis of randomized controlled trials". J Shoulder Elbow Surg 2017;26:79-80

6. Wali MG, Baba AN, Latoo IA, Bhat NA, Baba OK, Sharma S. Internal fixation of shaft humerus fractures by dynamic compression plate or interlocking intramedullary nail: a prospective, randomised study. Strategies Trauma Limb Reconstr 2014;9:133-40.

7. Mückley T, Diefenbeck M, Sorkin AT, Beimel C, Goebel M, Bühren V. Results of the T2 humeral nailing system with special focus on compression interlocking. Injury 2008;39:299-305

8. Herbst U, Ruettger K, Mockwitz J. Experiences with the Russell-Taylor Nail in Humeral Shaft Fractures-An Analysis of Postoperative Results and Complications. Osteo Trauma Care 2003;11:13-20.

9. Franck WM, Olivieri M, Jannasch O, Hennig FF. Expandable nail system for osteoporotic humeral shaft fractures: preliminary results. J Trauma 2003; $54: 1152-8$.

10. Persiani P, Gurzi M, Moreschini O, Di Giacomo G, Villani C. Fluoroscopic freehand and electromagnetic-guided targeting system for distal locking screws of humeral intramedullary nail. Musculoskeletal Surg 2017:101:19-23.

11. Choudhari P, Baxi M, Patidar S. Fracture Shaft Humerus: Plating OR Nailing? Orthopaedic Journal of MP Chapter 2016:22.

12. Kulkarni VS, Kulkarni MS, Kulkarni GS, Goyal V, Kulkarni MG Comparison between antegrade intramedullary nailing (IMN), open reduction plate osteosynthesis (ORPO) and minimally invasive plate 
osteosynthesis (MIPO) in treatment of humerus diaphyseal fractures. Injury 2017;48(Suppl 2):8-13.

13. Berkes MB, Little MT, Lorich DG. Complications of Intramedullary Nailing, in Intramedullary Nailing. Springer 2015:85-94.

14. Noda M, Saegusa Y, Maeda T. Does the location of the entry point affect the reduction of proximal humeral fractures? A cadaveric study. Injury 2011;42(Suppl 4):35-8.

15. Shah SM, Ajgaonkar AR. Diaphyseal fractures of humerus: intramedullary nail versus plate fixation. Bombay Hosp J 2012;54:51-7.

16. Jang Y, Kempton LB, Mckinley TO, Sorkin AT. Insertion-related pain with intramedullary nailing. Injury 2017;48(Suppl 1):18-21.

17. Campochiaro G, Baudi P, Loschi R, Serafin F, Catani F. Complex fractures of the humeral shaft treated with antegrade locked intramedullary nail: clinical experience and long-term results. Acta Biomed 2015;86:69-76.

18. Rajagopal HP,Mohan MM, Pilar A, Tamboowalla Behram K. Functional outcome of antegrade interlocking intramedullary nailing for humeral shaft fractures. Int J Res Orthop 2017;3:1127-31.
19. Kulkarni VS, Kulkarni MS, Kulkarni GS, Goyal V, Kulkarni MG. Comparison between antegrade intramedullary nailing (IMN), open reduction plate osteosynthesis (ORPO) and minimally invasive plate osteosynthesis (MIPO) in treatment of humerus diaphyseal fractures. Injury 2017;48:813.

20. Changulani M, Jain UK, Keswani T. Comparison of the use of the humerus intramedullary nail and dynamic compression plate for the management of diaphyseal fractures of the humerus. A randomised controlled study. Int Orthop 2007;31:391-5.

21. Barber FA, Fischer SP. muz ve Dirsek İçin Cerrahi Girișimler. Içinde: Esenyel CZ, Mahiroğulları M, Çakmak S. OHabitat Yayınevi, 2013:3-6.

22. Marecek GS, Saltzman MD. Complications in shoulder arthroscopy. Orthopedics 2010;33:492-497.

23. Weber SC, Abrams JS, Nottage WM. Complications associated with arthroscopic shoulder surgery. Arthroscopy 2002;18: 88-95. 